\title{
Engineering Polymeric Prodrug Nanoplatform for Vaccination Immunotherapy of Cancer
}

Lei Zhou ${ }^{1,2, \#, \text { Bo Hou }}{ }^{1, \#}$, Dangge Wang ${ }^{1}$, Fang Sun ${ }^{1}$, Rundi Song ${ }^{1}$, Qian Shao ${ }^{3}$, Hao Wang ${ }^{2}$, Haijun $\mathrm{Yu}^{1,4, *}$, Yaping $\mathrm{Li}^{1,4, *}$

1. State Key Laboratory of Drug Research \& Center of Pharmaceutics, Shanghai Institute of Materia Medica, Chinese Academy of Sciences, Shanghai 201203, China;

2. China State Institute of Pharmaceutical Industry, Shanghai 201203, China;

3. Shandong Institute of Cancer Prevention and Treatment, Jinan, 250117, China;

4. Yantai Key Laboratory of Nanomedicine \& Advanced Preparations, Yantai Institute of Materia Medica, Shandong 264000, China.

\#: Contribution equally to this study.

[*] Corresponding authors: Prof. Haijun Yu, hjyu@simm.ac.cn, Prof. Yaping Li, ypli@simm.ac.cn, Tel/Fax: +86-21-2023-1979.

State Key Laboratory of Drug Research \& Center of Pharmaceutics, Shanghai Institute of Materia Medica, Chinese Academy of Sciences, Shanghai 201203, China; Yantai Key Laboratory of Nanomedicine \& Advanced Preparations, Yantai Institute of Materia Medica, Shandong 264000, China. 


\section{Table of Contents}

Materials and Methods

Page 3-14

Figure S1.

Page 15

Figure S2.

Page 16

Figure S3.

Page 17

Figure S4.

Page 18

Figure S5.

Page 19

Figure S6.

Page 20

Figure S7.

Page 21

Figure S8.

Page 22

Figure S9.

Page 23

Figure S10.

Page 24

Figure S11.

Page 25

Figure S12.

Page 26

Figure S13.

Page 27

Figure S14.

Page 28

Figure S15.

Page 29 


\section{Experimental Section}

Materials. 4-(hydroxymethyl) phenol, dichloromethane (DCM, anhydrous), 2-(7Azabenzotriazol-1-yl)-N, N, N', N'-tetramethyluronium hexafluorophosphate (HATU), methacryloyl chloride, 5,6-Dimethyl-9-oxo-9H-xanthene-4-acetic acid (DMXAA), 4Cyano-4-(dodecylsulfanylthiocarbonyl) sulfanylpentanoic acid (CTA), 1-(3Dimethylaminopropyl)-3-ethylcarbodiimide Hydrochloride (EDCI), $\mathrm{mPEG}_{113}-\mathrm{NH}_{2}$, $\mathrm{NH}_{2}-\mathrm{PEG}_{113}-\mathrm{NH}_{2}$, diisopropylamino ethyl methacrylate (DPA), oligoethylenimine

(OEI) 800 Da, 1,2-Epoxytetradecane, 3-(4,5-dimethylthiazol-2-yl)-2,5diphenyltetrazolium bromide (MTT) and lipopolysaccharide (LPS) were all ordered from Sigma-Aldrich China. Triethylamine (TEA), hydroxyl benzotrizole (HOBT), N, N-Dimethylformamide (DMF), azodiisobutyronitrile (AIBN) were purchased from J\&K Chemical. $\alpha$-D-Mannopyranosylphenyl isothiocyanate was obtained from Beijing Chemsynlab Pharmaceutical Science \& Technology Co. Ltd. Hoechst 33342, 4',6diamidino-2-phenylindole (DAPI) and LysoTracker Red (DND-99) were order from Life Technologies (Carlsbad, CA). Dialysis tubing (MWCO 3500 Da) was obtained from Fisher Scientific. Inc. (IL, USA). Other chemical and reagents not mentioned above were all in analytical grade and purchased from Sinopharm Group Chemical Reagent Co., Ltd. 
OVA (SIINFEKL), OVA-FITC and M32 (SHRSCSHQTSAPSPKALAHNGTPR NAI) were custom ordered from BankPeptide Biological Technology Co., Ltd. Antimouse PD-L1 antibody ( $\alpha$ PDL1) was purchased from Bioxcell (Ohio, USA). ELISA kit of TNF- $\alpha$, IFN- $\gamma$, iL-6, iL-12p40 and IFN $\beta$ were all purchased from Neobioscicence Technology Co., Ltd. The mouse lymphocyte separation medium was obtained from Dakewe Biotech, China. Antibodies against CD11c, CD80, CD80, CD45, CD3, CD4, CD8, IFN- $\gamma$, Foxp3, CD44, CD127, MHC II, CD11b, F4/80 and B220 for flow cytometry were all obtained from BD Biosciences. Antibodies against GAPDH, phosphorylated TBK1 and IRF3 for western blot were all ordered from CST. H-2Kb OVA Tetramer-SIINFEKL and CD8 (clone: KT15) were purchased from Medical \& Biological Laboratories (MBL) Co., Ltd. Trizol reagent, Hifair ${ }^{\circledR}$ II 1st Strand cDNA Synthesis SuperMix (Cat:11120ES60) and Hieff UNICON® qPCR SYBR Green Master Mix (Cat:11198ES08) were all obtained from Yeasen (Shanghai, China). The primer of mIFN- $\beta$, mCXCL10, mIsg15 and mGAPDH were all custom ordered form Sangon Biotech Co., Ltd (Shanghai, China). OT-1 mice were provided by Shanghai Institute of Biochemistry and Cell Biology, CAS. Magnetic separation, anti-CD8 beads, Tissue Dissociator and smart strainer were obtained from MACS technology, Miltenyi Biotec (Germany).

Cell lines and animals. B16-OVA murine melanoma tumor cells were obtained from Sichuan University (Chengdu, China). 4T1 murine breast tumor cells were purchased from the Cell Bank of Chinese Academy of Science (Shanghai, China). BMDCs derived from bone marrow monocytes were flushed from the femurs of C57BL/6 mice, 
and were stimulated with $20 \mathrm{ng} / \mathrm{mL}$ GM-CSF and $10 \mathrm{ng} / \mathrm{mL}$ iL-4 for 6 days. Then nonadherent and loosely adherent BMDCs were collected on day- 6 for future use. All cells were cultured in RPMI 1640 medium with $10 \% \mathrm{v} / \mathrm{v}$ FBS, $100 \mathrm{U} / \mathrm{mL}$ of penicillin and $100 \mu \mathrm{g} / \mathrm{mL}$ of streptomycin at $37^{\circ} \mathrm{C}$ in $5 \% \mathrm{CO}_{2}$.

Four-week old BALB/c mice and C57BL/6 mice (18-20 g, female) were supplied by Shanghai Experimental Animal Center (Shanghai, China). All animal procedures were carried out under the guidelines approved by the Institutional Animal Care and Use Committee (IACUC) of Shanghai Institute of Material Medica, Chinese Academy of Sciences.

Synthesis of OEI-C14 and acid-responsive diblock copolymers. Alkylated OEI-800 (OEI-C14) was synthesized by reacting OEI-800 with 1,2-epoxytetradecane and purified by following our previously reported procedure. ${ }^{1}$ To synthesize DMXAAgrafted PEG- $b$-PDPA diblock copolymer, 4-hydroxybenzyl methacrylate was first prepared by the following method: 4-(hydroxymethyl) phenol (192.5 mg, $1.00 \mathrm{mmol})$ and TEA (303.5 mg, $3.00 \mathrm{mmol}$ ) were dissolved in anhydrous DCM and kept stirring under room temperature. Next, methacryloyl chloride (104.5 mg, $1.00 \mathrm{mmol})$ was added to the above solution and kept reaction under argon protection at room temperature. The solution was condensed by vacuum evaporation $24 \mathrm{~h}$ post reaction. The crude product was purified by silica gel chromatography with a gradient eluent of DCM and methanol $(\mathrm{V}: \mathrm{V}=40: 1-10: 1)$.

The obtained 4-hydroxybenzyl methacrylate $(288.3 \mathrm{mg}, 1.50 \mathrm{mmol})$ was then reacted with DMXAA $(282.5 \mathrm{mg}, 1.00 \mathrm{mmol})$, HATU (1.140 g, $3.0 \mathrm{mmol})$ and TEA 
(303.5 mg, $3.00 \mathrm{mmol}$ ) in anhydrous DCM under ice bath. The reaction was continued for $24 \mathrm{~h}$ and the final product was purified by silica gel chromatography using a gradient eluent of dichloromethane and methanol $(\mathrm{V}: \mathrm{V}=80: 1-20: 1)$. The obtain DMXAA methacrylate was characterized by ${ }^{1} \mathrm{H}-\mathrm{NMR}$ spectra.

To synthesize the macromolecular chain transfer agent PEG $_{113}$-CTA, CTA (19.3 mg, $0.048 \mathrm{mmol})$, EDCI (27.5 mg, $0.144 \mathrm{mmol})$ and HOBT (19.4 mg, $0.144 \mathrm{mmol})$ were dissolved in anhydrous DMF under stirring for $1.5 \mathrm{~h}$. Next, $\mathrm{mPEG}_{113}-\mathrm{NH}_{2}(200.8$ $\mathrm{mg}, 0.040 \mathrm{mmol})$, TEA $(16.16 \mathrm{mg}, 0.160 \mathrm{mmol})$ were added into the solution under argon protection at ice bath. After $24 \mathrm{~h}$ reaction, the reactants were dialyzed against with ethanol and DI water, and lyophilized for further use.

$\mathrm{NH}_{2}-\mathrm{PEG}_{113}-\mathrm{CTA}$ was synthesized by following the above method. CTA (19.7 mg, $0.048 \mathrm{mmol})$, EDCI (28.1 mg, $0.144 \mathrm{mmol})$ and HOBT (19.8 mg, $0.144 \mathrm{mmol})$ were all dissolved in anhydrous DMF. $\mathrm{NH}_{2}-\mathrm{PEG}_{113}-\mathrm{NH}_{2}(201.2 \mathrm{mg}, 0.040 \mathrm{mmol})$ and TEA (16.2 $\mathrm{mg}, 0.160 \mathrm{mmol}$ ) were added $1.5 \mathrm{~h}$ post reaction. The reaction was stopped post $24 \mathrm{~h}$ and further purified by dialysis against DI water to obtain target product.

$\mathrm{mPEG}_{113}-b-\mathrm{P}\left(\mathrm{DPA}_{40}-\mathrm{co}-\mathrm{DMXAA}_{10}\right)$ copolymer was synthesized by RAFT polymerization. Briefly, $\mathrm{mPEG}_{113}$-CTA (100.4 mg, $\left.0.019 \mathrm{mmol}\right)$, DPA (237.3 mg, $1.114 \mathrm{mmol})$, DMXAA methacrylate $(260.2 \mathrm{mg}, 0.296 \mathrm{mmol})$ and AIBN (0.31 mg, $0.0019 \mathrm{mmol}$ ) were dissolved in $1.0 \mathrm{~mL}$ of anhydrous DMF, following with three-cycle of freezing and thawing to remove oxygen inside. Then the reaction was continued for $24 \mathrm{~h}$ at $70{ }^{\circ} \mathrm{C}$ oil bath. The reactant was dialyzed against DI water and lyophilized to obtain the final product. 
To synthesize Man-functionalized $\mathrm{PEG}_{113}-\mathrm{PDPA}_{50}$ diblock copolymer, $\mathrm{NH}_{2}$ $\mathrm{PEG}_{113}-\mathrm{PDPA}_{50}$ copolymer was first prepared by $\mathrm{NH}_{2}-\mathrm{PEG}_{113}-\mathrm{CTA}$-initialized RAFT polymerization of DPA. Then $\mathrm{NH}_{2}-\mathrm{PEG}_{5 \mathrm{~K}}-\mathrm{PDPA}_{40}(160.3 \mathrm{mg}, 0.001 \mathrm{mmol}), \alpha-\mathrm{D}-$ mannopyranosylphenyl isothiocyanate $(1.57 \mathrm{mg}, 0.005 \mathrm{mmol})$ and DIEA (0.645 $\mathrm{mg}$, $0.005 \mathrm{mmol}$ ) were reacted in anhydrous DMF for $24 \mathrm{~h}$ at room temperature. The final product was obtained by dialyzing against ethanol and DI water. The structure was examined by ${ }^{1} \mathrm{H}-\mathrm{NMR}$ spectrum.

Esterase-triggered DMXAA release. PDPM was dissolved in DMSO and diluted in methanol with a final concentration of $20 \mathrm{mg} / \mathrm{mL}$. Then $20 \mu \mathrm{L}$ PDPM solution was added to $100 \mu \mathrm{L}$ of porcine liver esterase solution $(120 \mathrm{U} / \mathrm{mL})$. The obtained mixture was incubated with different time points at $37^{\circ} \mathrm{C}$. At time intervals, $100 \mu \mathrm{L}$ mixture was added with $400 \mu \mathrm{L}$ methanol and then centrifuged at $8000 \mathrm{rpm}$ for $10 \mathrm{~min}$ to collect the supernatant for HPLC analysis.

Fabrication and characterization of nanovaccine. To fabricate neoantigen-loaded nanovaccine, polymeric micelle nanoparticles were firstly prepared by a nanoprecipitation method. Briefly, the diblock copolymer and OEI-C14 were dissolved in tetrahydrofuran (THF) at indicated weight ratios and then added into DI water under ultrasonication. THF was removed by dialyzed against DI water overnight to obtain the desired micelle nanoparticles.

To prepare the nanovaccine, peptides were first dissolved in DMSO at $10 \mathrm{mg} / \mathrm{mL}$ and then dispersed in endotoxin-free water. The copolymers were dissolved in THF and then dropped into peptide solution under ultrasonication. Subsequently, THF and free 
peptides were removed by ultrafiltration for $15 \mathrm{~min}$ (molecular weight cut-off $50 \mathrm{kDa}$ ).

To determine the peptide loading efficiency, the supernatant containing peptideloaded micelle nanoparticles were collected and dissociated in methanol. The percentage of nanoparticles-loaded peptides was measured by HPLC.

To determine the hemolytic property of the micelle nanoparticles, the nanoparticles were incubated with erythrocytes in different $\mathrm{pH}$ buffer (i.e., $\mathrm{pH}=7.4$, $6.4,6.0)$ at $37{ }^{\circ} \mathrm{C}$ for $1 \mathrm{~h}$. The positive control group for complete erythrocytes lysis was obtain by red lysis buffer. Finally, the hemoglobin release was measured by UVvis microplate reader $(\mathrm{Abs}=450 \mathrm{~nm})$.

To evaluate safety and biocompatibility of various nanoparticle formulations in vitro, DC2.4 cells were plated into 96 -well dishes at a density of $4 \times 10^{3}$ cells per well for $24 \mathrm{~h}$. Then cells were incubated with the micelle nanoparticles at indicated concentration for $48 \mathrm{~h}$. The cell viability was examined by MTT assay.

Nanovaccine uptake with the BMDCs in vitro and in vivo. In vitro cellular uptake of the nanovaccines with BMDCs was investigated by flow cytometric examination. Briefly, BMDCs freshly collected from BALB/c mice were seeded in 24-well plates at a density of $5 \times 10^{5}$ cells per well for $24 \mathrm{~h}$, and incubated with OVAFITC-loaded nano vaccines (e.g. PDPE@OVA, Man-PDPE@OVA) at the desired time points $(2,4,6,8$, 10h). The intracellular fluorescence intensity of FITC was examined by flow cytometric measurement.

To investigate OVA uptake by APCs in vivo, various nanovaccines (NP: $50 \mathrm{mg} / \mathrm{kg}$, OVA: $2.5 \mathrm{mg} / \mathrm{kg}$ ) were administered subcutaneously at the tail base. Lymph nodes were 
resected $48 \mathrm{~h}$ post injection and digested into single cell suspensions. To recognize DCs uptake, cells were stained with anti-CD45-APC, anti-CD11c-PE, and anti-MHC IIpercpcy5.5 for detecting uptake of OVA antigen. Then the macrophages and B cells were stained with anti-CD45-APC, anti-CD11b-PE, anti-F4/80-PE-CY7 and antiCD45-APC, anti-B220-PE, respectively.

BMDC maturation and antigen-presentation in vitro. Immature BMDCs were obtained from BALB/c mice and seeded into 24 -well plates at $5 \times 10^{5}$ cells per well. The cells were stimulated with various nanoparticles $(100 \mu \mathrm{g} / \mathrm{mL})$ or nanovaccines (nanoparticles: $100 \mu \mathrm{g} / \mathrm{mL}$, OVA: $10 \mu \mathrm{g} / \mathrm{mL}$ ) for $24 \mathrm{~h}$. The treated BMDCs were then stained with anti-CD11c-FITC, anti-CD80-PE, and anti-CD86-PE-Cy7 for flow cytometric assessment. BMDCs treated with $1.0 \mu \mathrm{g} / \mathrm{mL}$ of LPS was set as the positive control.

To investigate the ability for antigen-presentation in vitro, DC2.4 cells were plated in 24-well dishes at a density of $1 \times 10^{6}$ cells per well and were allowed to attach overnight. Cells were treated with the desired nanovaccines for $6 \mathrm{~h}$ and then incubated with fresh medium for further $24 \mathrm{~h}$ or $48 \mathrm{~h}$. The cells were examined by flow cytometric measurement. Antigen-presentation in vitro was also investigated by CLSM measurement, nanoparticles loaded with FITC-labeled OVA was incubated with DC2.4 cells, following staining with Lysotracker RED (DND-99) for 40 min and Hoechst 33342 for $15 \mathrm{~min}$ in cell incubator. The cells were then washed with PBS for three times and fixed with 4\% paraformaldehyde prior to CLSM examination.

Cytokines secretion assay. To investigate different cytokines in vitro, cells were plated 
in 96-well dishes and pulsed with various treatments at desired time points. Next, the supernatant medium was collected and relevant cytokines production were quantified by ELISA kit according to manufacturer's protocols.

For quantifying intratumoral cytokine secretion, the tumors were resected on day-3 post immunization and dissociated into $0.5 \mathrm{~mL}$ of PBS per $0.5 \mathrm{~kg}$ tumor mass. The lysis concentration of TNF- $\alpha$, IFN- $\gamma$ and iL- 6 were analyzed by corresponding ELISA kit.

Real-Time PCR analysis. To perform RT-PCR analysis of STING activation, relevant mRNA was detected. BMDCs were firstly stimulated for $24 \mathrm{~h}$ and collected following with PBS wash for three times. Total RNA was extracted by trizol reagent from cells according to manufacturer's procedure. cDNA was synthesized by reverse transcription using Hifair ${ }^{\circledR}$ II 1st Strand cDNA Synthesis SuperMix. Finally, quantitative real-time PCR was performed using Hieff UNICON® qPCR SYBR Green Master Mix and relative quantification was calculated by the $2^{-\Delta \Delta \mathrm{Ct}}$ calculation normalized against GADPH. The primer sequences used for qPCR are listed as follows.

\author{
mIFN- $\beta$ : CTGGGTGGAATGAGACTATTGT (F); \\ AAGTTCCTGAAGATCTCTGCTC (R) \\ mCXCL10: CAACTGCATCCATATCGATGAC (F) \\ GATTCCGGATTCAGACATCTCT (R) \\ mIsg15: AGCGAGCCTCTGAGCATCCTG (F) \\ GCGTGTCTACAGTCTGCGTCAG (R) \\ mGAPDH: GGTTGTCTCCTGCGACTTCA (F) \\ TGGTCCAGGGTTTCTTACTCC (R)
}


T cell priming in vitro. BMDCs were stimulated with various nanovaccines (NP: 100 $\mu \mathrm{g} / \mathrm{mL}$, OVA: $10 \mu \mathrm{g} / \mathrm{mL}$ ) for $6 \mathrm{~h}$ following with fresh medium substitution for $24 \mathrm{~h}$ presentation. BMDCs were collected and washed in PBS for three times. Next, CD8 ${ }^{+}$ OT-1 T cells obtained from spleens of OT-1 mice were sorted by magnetic separation. Then pre-treated BMDCs were incubated with $\mathrm{CD} 8^{+} \mathrm{OT}-1 \mathrm{~T}$ cells in 96-well plates for further $24 \mathrm{~h}$ and the supernatant was collected for detecting IFN- $\gamma$ secretion by ELISA.

Biodistribution of nanovaccines in vivo. To investigate lymph node distribution of the nanovaccine, Ce6-labelled nanoparticle formulations (PDPE-Ce6, Man-PDPE-Ce6, PDPM-Ce6, or Man-PDPM-Ce6 at an identical Ce6 dose of $4 \mathrm{mg} / \mathrm{kg}$ ) were loaded with OVA $_{\text {FITC }}(2.5 \mathrm{mg} / \mathrm{kg})$ and injected in the tail base of C57BL/6 mice. The fluorescence images in vivo were acquired by IVIS imaging system (PerkinElmer, USA) at the desired time points. The inguinal and axillary LNs as well as the major organs were harvested at the desired time points for fluorescence imaging ex-vivo. The colocalization of the nanoparticles with OVA in the frozen sections of LNs was examined by using CLSM.

Antitumor study and prophylactic effect of nanovaccines. For antitumor efficacy assay, C57BL/6 mice were inoculated with $1 \times 10^{6}$ B16-OVA tumor cells on the right back. The mice were then randomly grouped into seven groups $(n=6$ in each group) when tumor volume reached around $50 \mathrm{~mm}^{3}$. The mouse groups were then treated with different formulations (i.e., 1: PBS, 2: DMXAA, $5 \mathrm{mg} / \mathrm{kg}, 3: \mathrm{OVA}, 2.5 \mathrm{mg} / \mathrm{kg}$, 4: PDPE, 50 mg/kg, 5: PDPE@OVA, 6: PDPM@OVA, Man-PDPM@OVA). The vaccination was repeated twice at an interval of a week. Animals were euthanized when tumor 
volume reached $1500 \mathrm{~mm}^{3}$ or exhibited cachexia and lethargy with heavy tumor burden. The tumor volume was monitored every other day and calculated by the following equation:

Tumor volume $=$ length $\times$ width $\times$ width $/ 2$ (length: the longest dimension, width: the shortest dimension).

In 4T1 tumor models, BALB/c mice were inoculated with 4T1 tumor cells in the right mammary gland. Accordingly, the antigens in the formulation were replaced with a 4T1-specific neoantigen M32. Other operations were as indicated above. As well, PDL1 antibody was administered intraperitoneally on day-1 and day-3 post each vaccination in the combination therapy.

To evaluate the prophylactic therapeutic efficacy, pre-vaccinated C57BL/6 mice were challenged with $1 \times 10^{6} \mathrm{~B} 16-\mathrm{OVA}$ tumors at the right back on day 7 after the third immunization. Tumor growth was monitored as indicated above.

Immune responses in vivo. To investigate nanovaccine-triggered immune responses in vivo, B16-OVA tumor bearing mice was vaccinated at tail base with varying formulations. The LNs were harvested 7-day post-vaccination and prepared into single cell suspensions. With immunofluorescence staining of anti-CD11c-FITC, anti-CD80PE, and anti-CD86-PE-CY7 for 30 min under room temperature, the frequency of DC maturation was examined by flow cytometry.

To verify the activation of antigen-specific $\mathrm{CD}^{+} \mathrm{T}$ cells, mouse blood and spleens were collected. Blood cells or splenocytes were stained with PE-tagged OVA-MHC tetramer and anti-CD8-FITC for $30 \mathrm{~min}$ under room temperature, followed with 
hemolysis and washing in PBS for three times prior to flow cytometric measurement.

For analysis of intratumoral infiltration of $\mathrm{T}$ lymphocytes, the tumor tissues were excised on day-7 post-vaccination and cut into small pieces, and processing with gentle MACS Dissociator with Tumor Dissociation Kit mouse according to the manufacture's procedure. The cell suspension was passed through a $70 \mu \mathrm{m}$ smart strainer following with $30 \mu \mathrm{m}$ smart strainer to acquire single cells. Then the lymphocytes were firstly enriched using the mouse lymphocyte separation medium. To determine the frequency of cytotoxic T lymphocytes (CTLs), the T lymphocytes were stained with anti-CD45APC, anti-CD3-percpcy5.5, anti-CD8-PE and anti-CD4-FITC. In parallel, the frequency of effector $\mathrm{T}$ cells were determined by anti-IFN- $\gamma$-FITC staining. $\mathrm{T}$ lymphocytes stained with anti-CD45-APC, anti-CD3-percpcy-5.5, anti-CD4-FITC and anti-Foxp3-PE were indicated for regulatory $\mathrm{T}$ cells.

To investigate the prophylactic immune responses, C57BL/6 mice were stimulated with nanovaccines for three times. DC maturation and antigen-specific $\mathrm{CD} 8^{+} \mathrm{T}$ cells in splenocytes were examined as indicated above. For immune memory effect assay, the spleen was excised on day-28 and analyzed by staining with immunofluorescence antibody (anti-CD8-FITC, anti-CD44-percpcy5.5 and anti-CD127-PE). The immune memory effect was further examined by incubating splenocytes from pre-vaccinated mice with free OVA ex-vivo for stimulation of antigen-specific memory T cells, and then the supernatant medium was harvested for detecting IFN- $\gamma$ by ELISA.

\section{Statistical Analysis}


Results are given as Mean \pm S.D. One way analysis of variance (ANOVA) was used to determine the significance of the difference. Statistical significance was set at ${ }^{*} p<0.05$, $* * \mathrm{p}<0.01, * * * \mathrm{p}<0.001, * * * * \mathrm{p}<0.0001$

\section{Reference}

(1) Wang, D.; Wang, T.; Liu, J.; Yu, H.; Jiao, S.; Feng, B.; Zhou, F.; Fu, Y.; Yin, Q.; Zhang, P.; Zhang, Z.; Zhou, Z.; Li, Y. Nano Lett. 2016, 16, 5503-5513. 
a
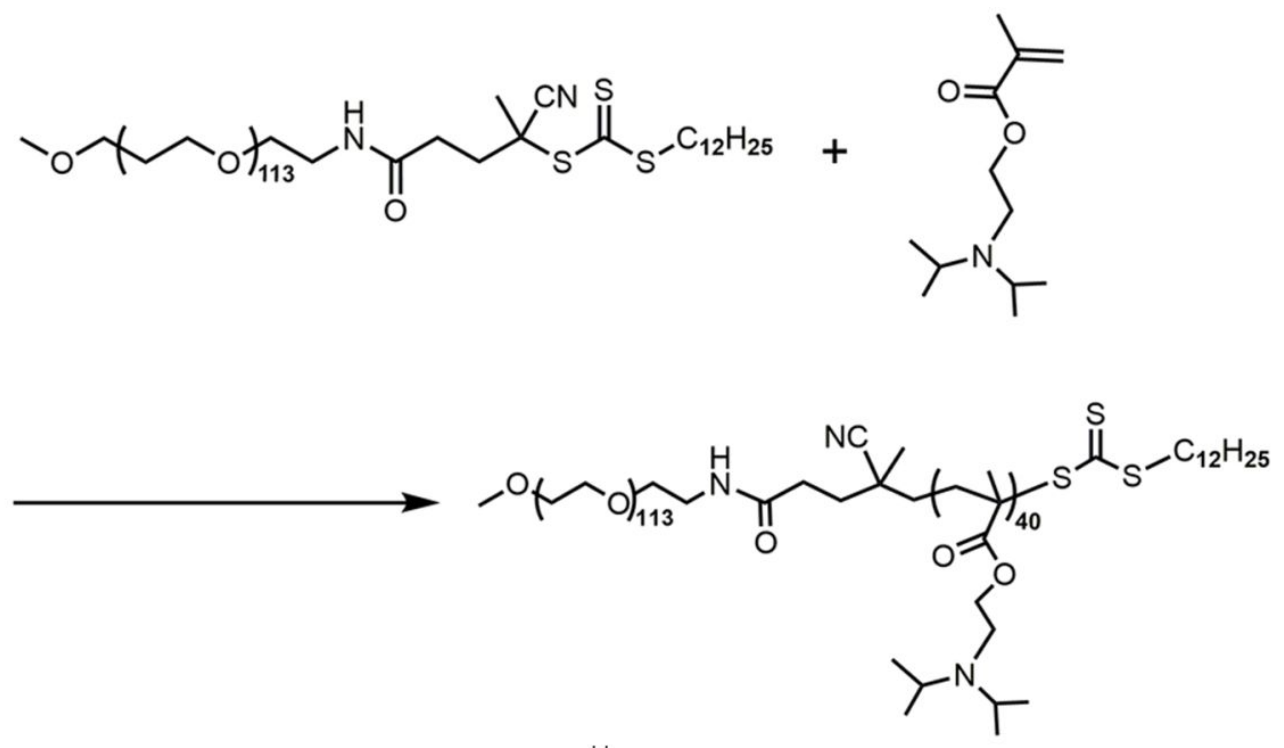

b

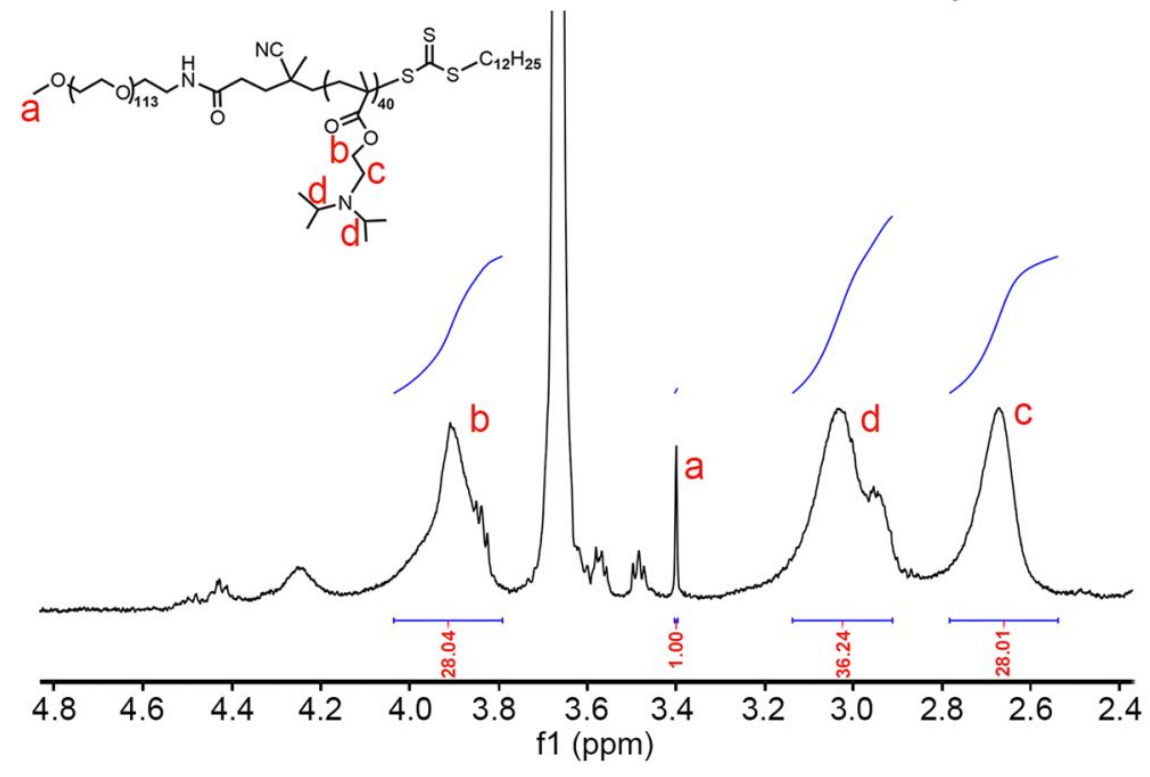

Figure S1. Characterization of $\mathbf{m P E G}_{113}-b-\mathrm{PDPA}_{40}$ diblock copolymer. (a) Synthesis route of $\mathrm{mPEG}_{113}-b-\mathrm{PDPA}_{40}$ diblock copolymer via RAFT polymerization; (b) ${ }^{1} \mathrm{H}-\mathrm{NMR}$ spectrum of $\mathrm{mPEG}_{113}-b$-PDPA 40 diblock copolymer. 
a<smiles>C=C(C)C(=O)OCc1ccc(O)cc1</smiles><smiles>Cc1ccc2c(=O)c3cccc(CC(=O)O)c3oc2c1C</smiles>

b<smiles>C=C(C)C(=O)OCc1ccc(OC(=O)Cc2cccc3c(=O)c4ccc(C)c(C)c4oc23)cc1</smiles>

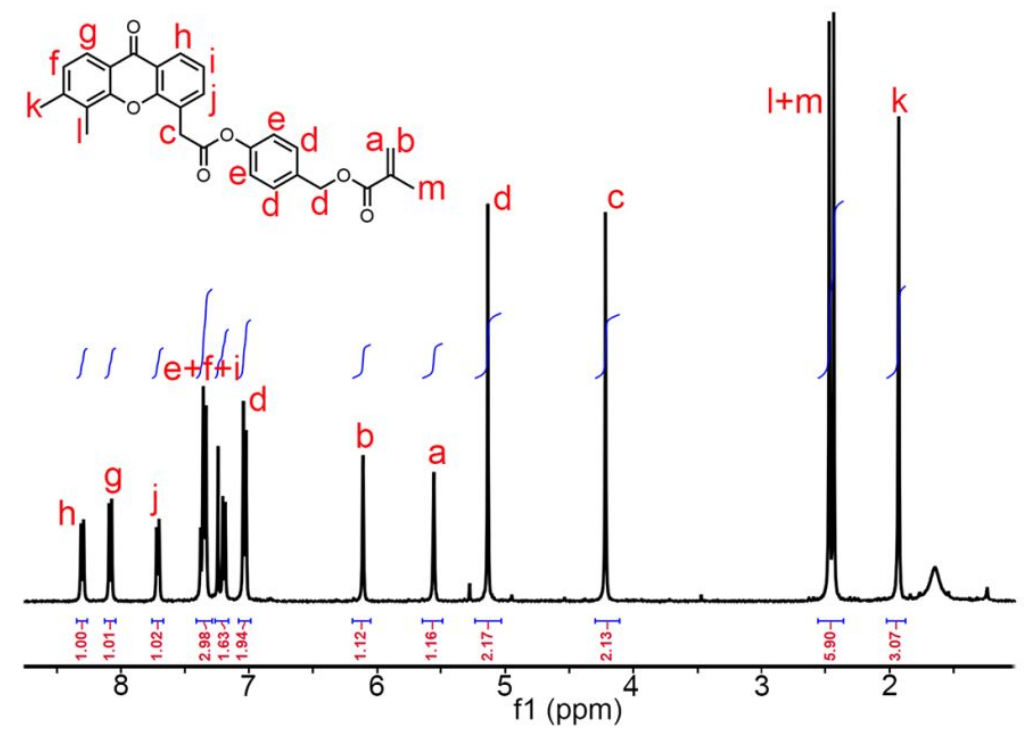

Figure S2. Characterization of DMXAA methacrylate. (a) Synthesis route of DMXAA methacrylate, and (b) ${ }^{1} \mathrm{H}-\mathrm{NMR}$ spectra of DMXAA methacrylate. 

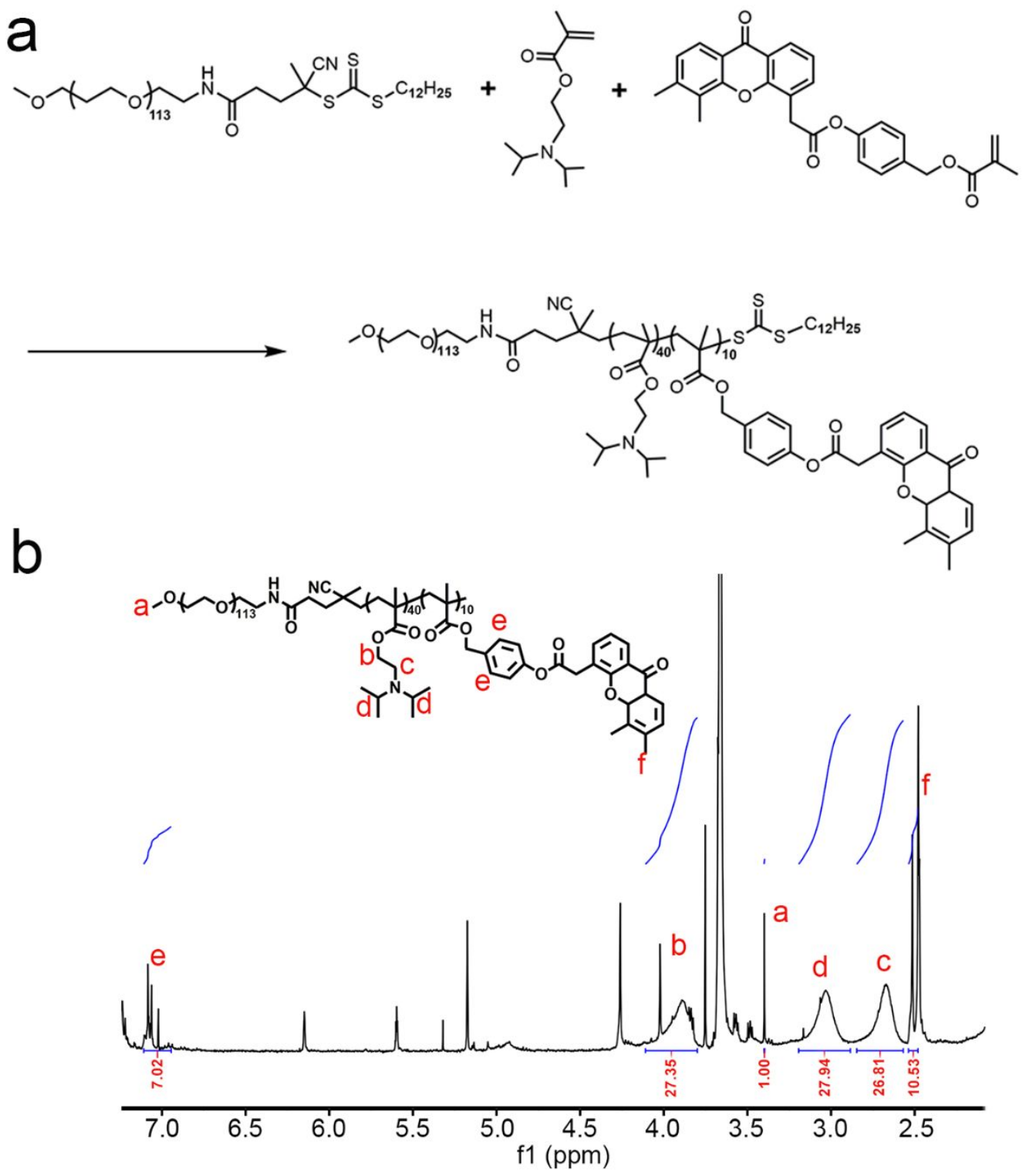

Figure S3. Synthesis and ${ }^{1} \mathrm{H}-\mathrm{NMR}$ characterization of DMXAA-grafted $\mathrm{mPEG}_{113^{-}}$ b-P(DPA40-co-DMXAA A $\left._{10}\right)$ diblock copolymer. (a) Synthetic route of $\mathrm{mPEG}_{113}-b$ $\mathrm{P}\left(\mathrm{DPA}_{40}-\mathrm{co}-\mathrm{DMXAA}_{10}\right)$, and (b) ${ }^{1} \mathrm{H}-\mathrm{NMR}$ spectrum of $\mathrm{mPEG}_{113}-b-\mathrm{P}\left(\mathrm{DPA}_{40}-\mathrm{co}-\right.$ DMXAA $_{10}$ ). 

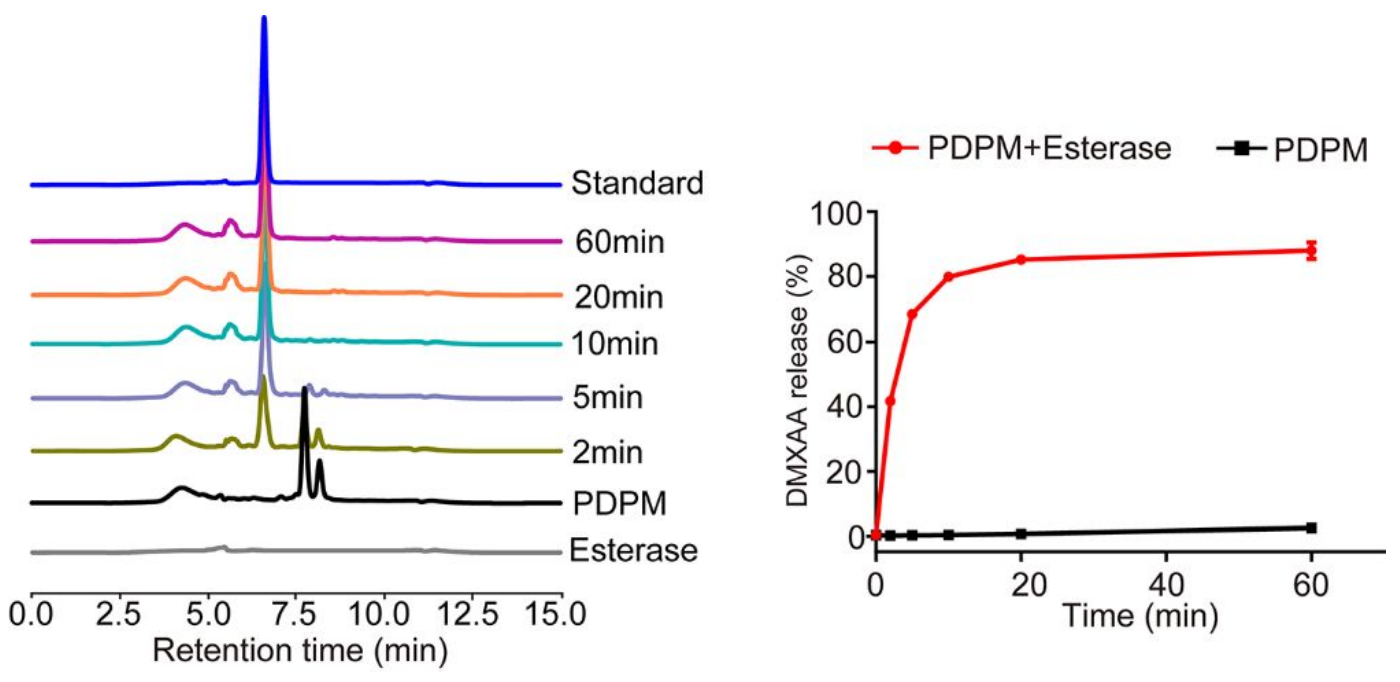

Figure S4. HPLC examination of esterase-mediated DMXAA release from PDPM and the release profile of PDPM. 
a
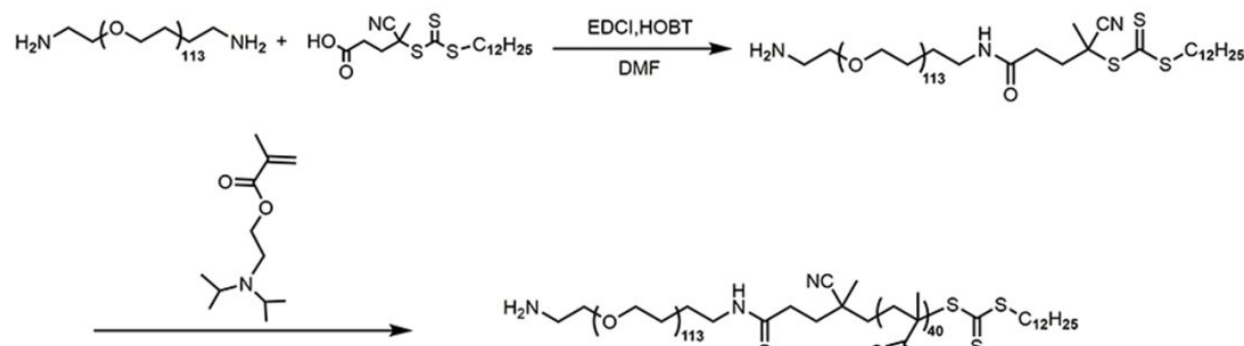

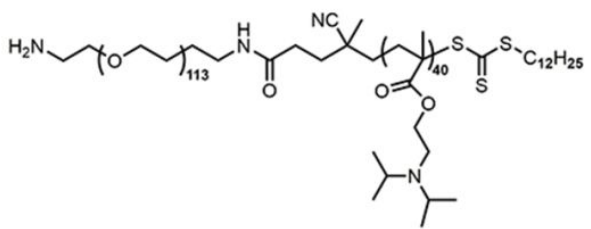
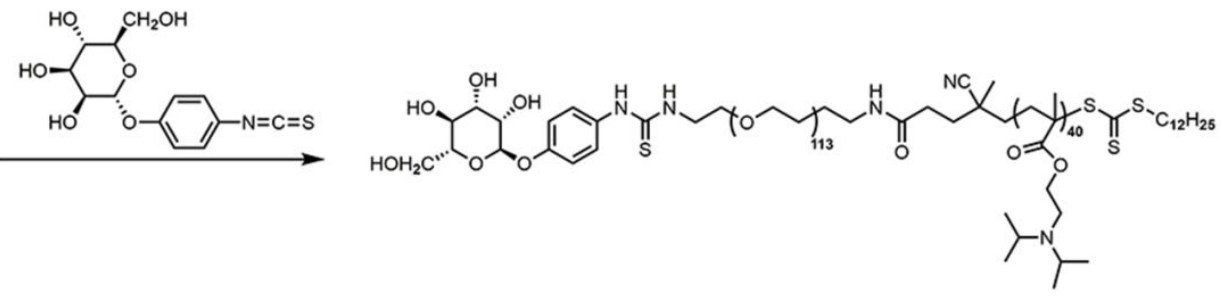

$\mathrm{b}$

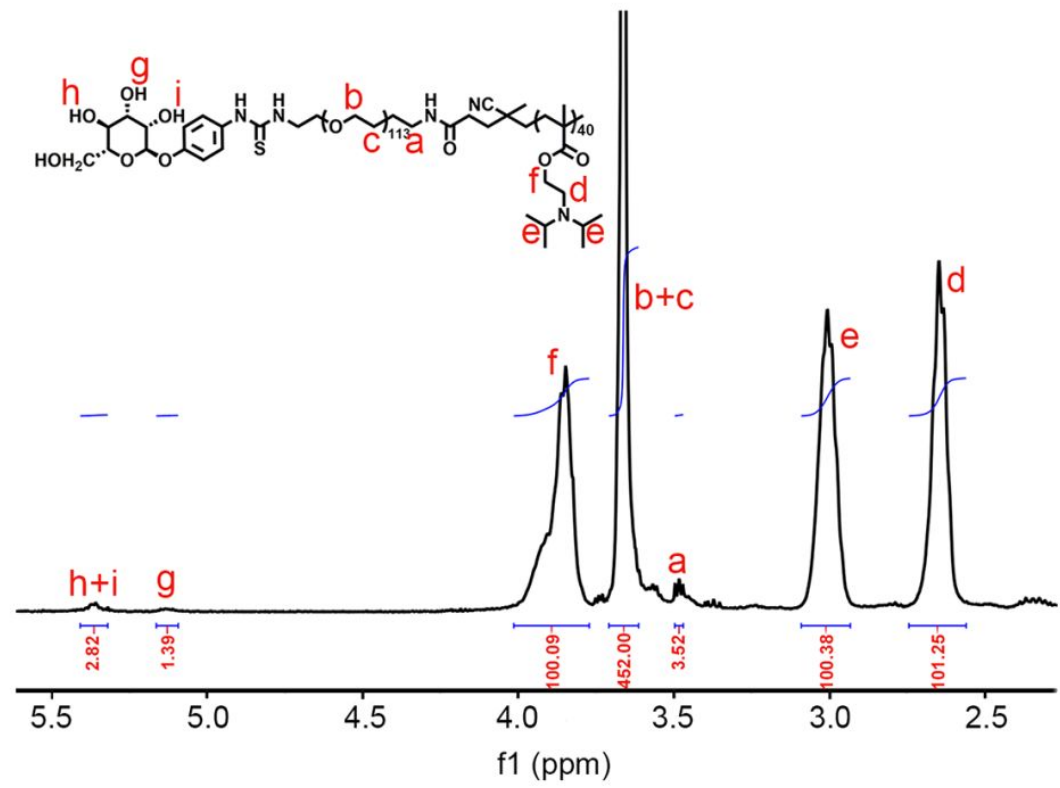

Figure S5. Characterization of Man-PEG $\mathbf{1 1 3}_{\mathbf{1 3}} \boldsymbol{b}-\mathbf{P D P A}_{\mathbf{4 0}}$. (a) The synthetic route and

(b) ${ }^{1} \mathrm{H}-\mathrm{NMR}$ spectrum of Man-PEG ${ }_{113}-b-\mathrm{PDPA}_{40}$. 

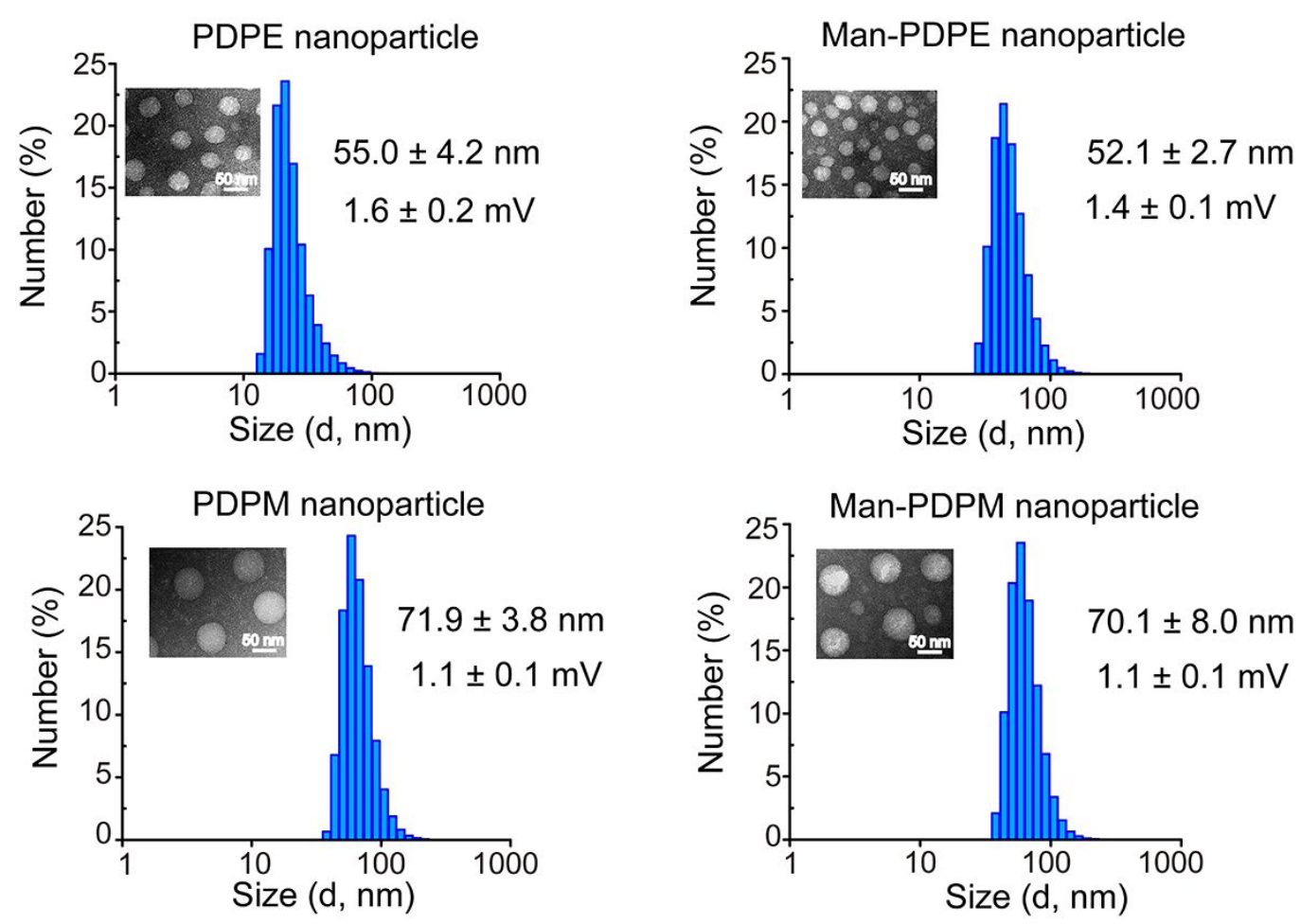

Figure S6. Size distribution and TEM images of PDPE, Man-PDPE, PDPM and ManPDPM nanoparticles. (scale bar $=50 \mathrm{~nm})$; 
a

$\square$ PDPA $\square$ PDPE (9:1)

$\square$ PDPA/PEI (7:3) $\square$ PDPA/PEI (5:5)

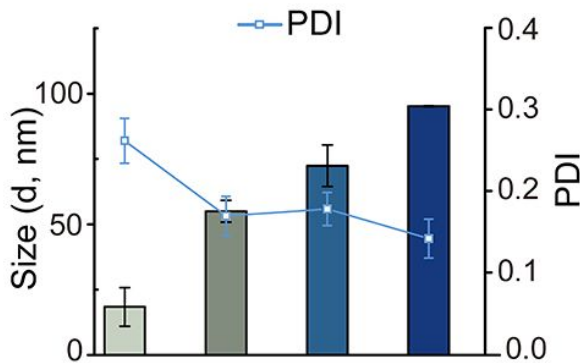

C

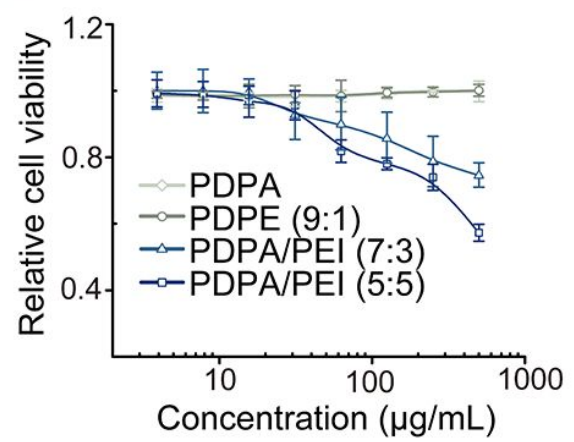

b
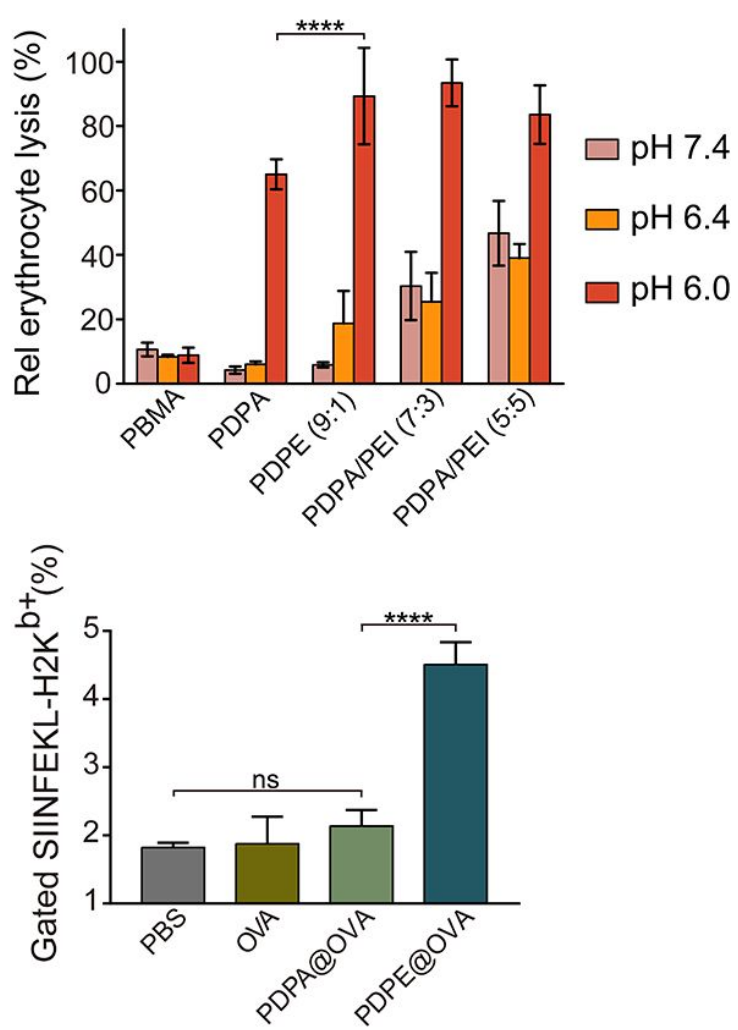

Figure S7. Physicochemical characterization of various nanoparticles. (a) Size of nanoparticles with different PDPA and PEI mass ratio measured by DLS; (b) Hemolysis analysis of nanoparticles at different $\mathrm{pH}$ buffer. PBMA is nanoparticle without $\mathrm{pH}$ responsive property; (c) Cytotoxic assay of DCs treated with indicated formulations; (d) Analysis of antigen-presentation after stimulated with different formulations. 

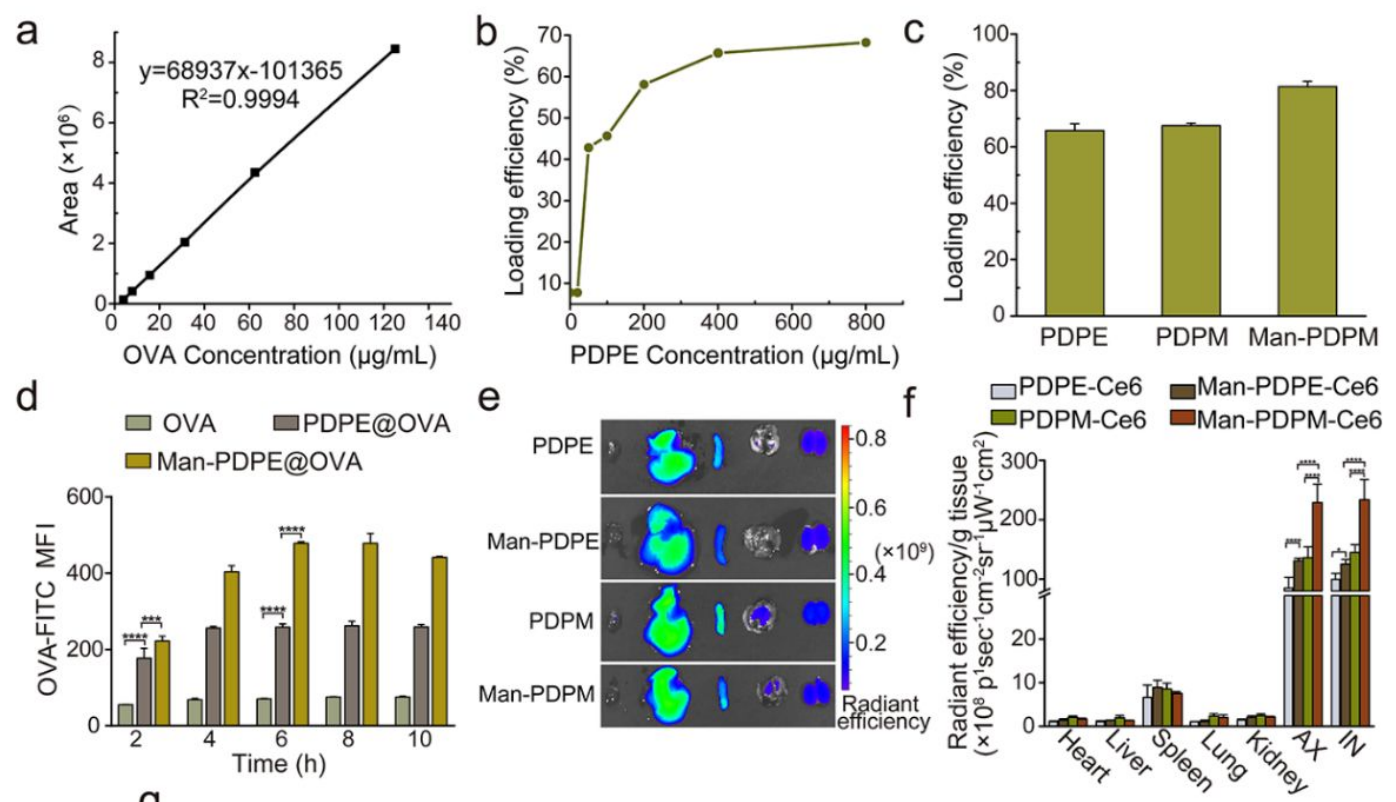

9
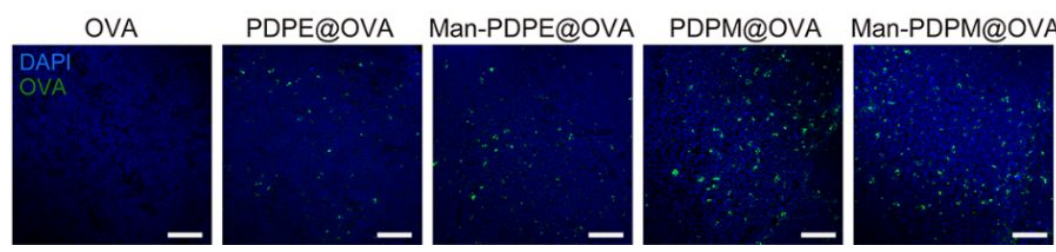

Figure S8. The loading and delivery capacity of OVA in vitro and in vivo. (a) The standard curves of free OVA determined by HPLC; (b) The OVA $(100 \mu \mathrm{g} / \mathrm{mL})$ loading efficiency in nanoparticles with various concentration $(0,20,50,100,200,400,800$ $\mu \mathrm{g} / \mathrm{mL}$ ); (c) OVA loading efficiency with different nanoparticles (OVA: $100 \mu \mathrm{g} / \mathrm{mL}$, nanoparticles: $400 \mu \mathrm{g} / \mathrm{mL}$ ); (d) FITC-tagged OVA was loaded in nanoparticles with or without mannose modification. BMDCs were treated with nano vaccines at various time points and mean fluorescence intensity of OVA-FITC was detected for cellular uptake capability; (e) Representative fluorescence images of the major organs (from left to right: heart, liver, spleen, lung and kidney) examined 7-day post administration; (f) biodistribution of nanovaccines in major organs and LNs 7-days post administration; (g) OVA accumulation in LNs $48 \mathrm{~h}$ post subcutaneous administration. LNs were harvested and frozen for observation (scale bar $=100 \mu \mathrm{m})$. 

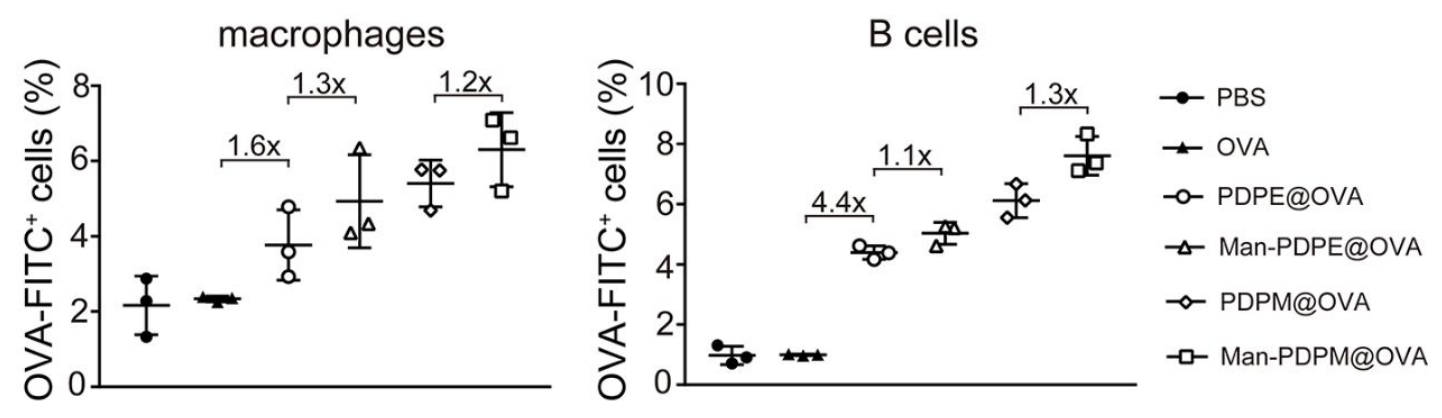

Figure S9. Flow cytometric analysis of OVA uptake by macrophages and B cells in vivo $48 \mathrm{~h}$ after s.c. administration. 

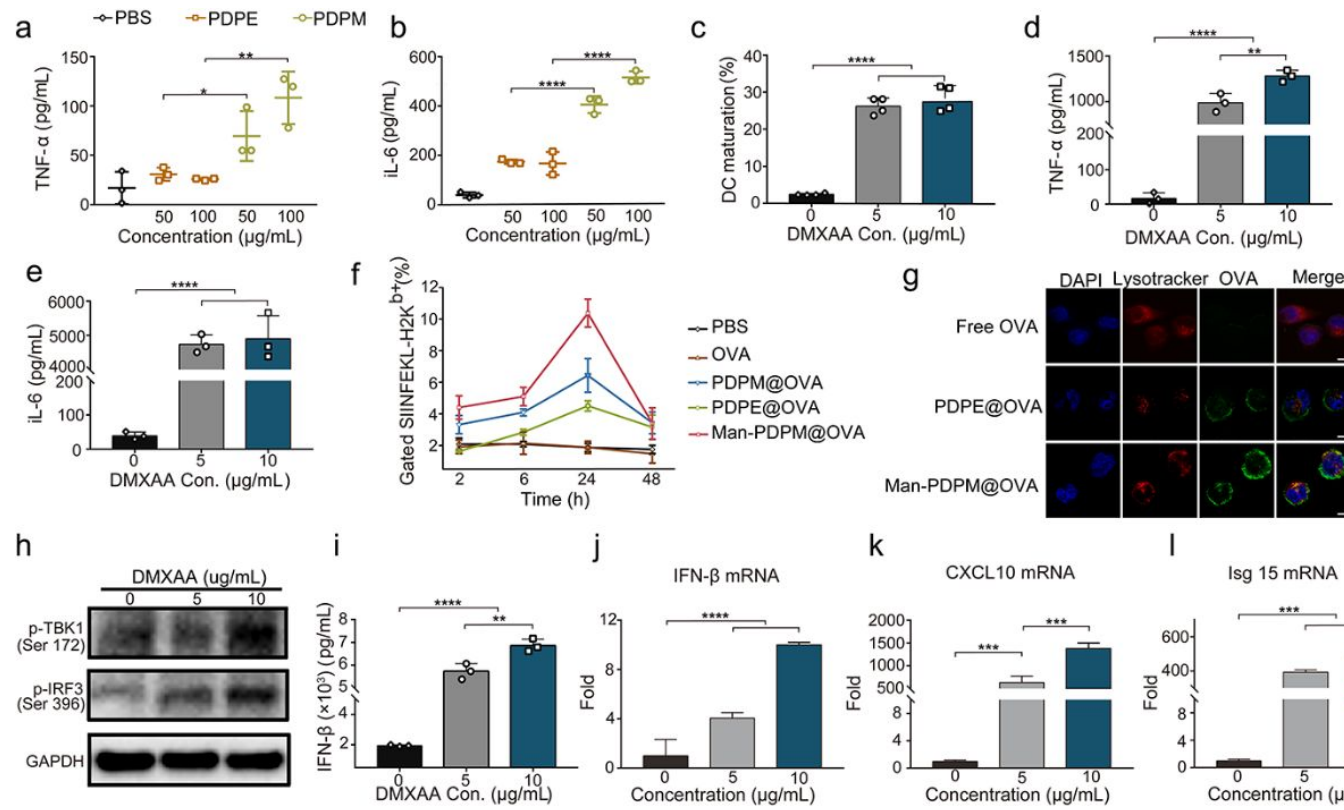

j

IFN- $\beta$ mRNA

$\sim$ PBS

COVA

-PDPE@OVA

-Man-PDPM@OVA

g

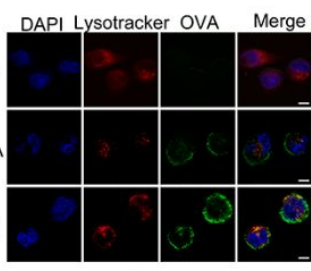

Man-PDPM@OVA

k

CXCL10 mRNA
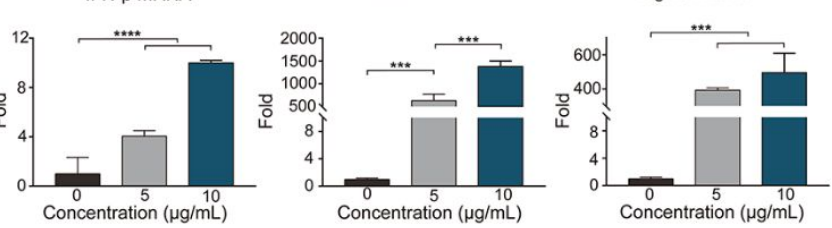

Figure S10. The promoted effect of antigen-presentation induced by STING

pathway activation. (a \& b) ELISA analysis of (a) TNF- $\alpha$ and (b) iL-6 production in the supernatant medium with nanoparticles treatment; (c) The frequency of DC maturation induced by DMXAA directly. BMDCs were inoculated with DMXAA at different concentration for $24 \mathrm{~h}$, following by flow cytometry assessment. (Gated on

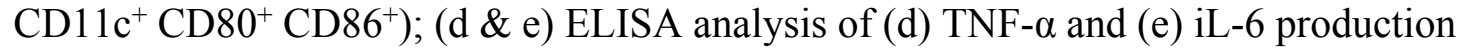
in the supernatant medium with DMXAA stimulation; (f) Antigen presentation of DCs pulsed with various formulations at indicated time points; (g) Confocal microscopy images of DCs incubated with nanovaccines at $6 \mathrm{~h}($ scale bar $=7.5 \mu \mathrm{m}) ;(\mathrm{h})$ Phosphorylation of TBK1 and IRF3 in the STING pathway indicated by western blot; (i) Secretion of IFN $\beta$ with DMXAA stimulation. BMDCs were seeded in 96-well plates with DMXAA for $24 \mathrm{~h}$, and the supernatant medium was collected for ELISA analysis; (j-1) mRNA expression level of (j) IFN- $\beta$ (k) CXCL 10 and (1) Isg-15 measured by RTPCR and normalized by GAPDH. 


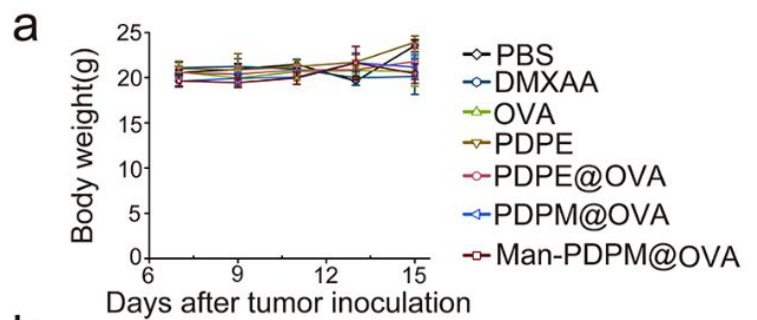

b

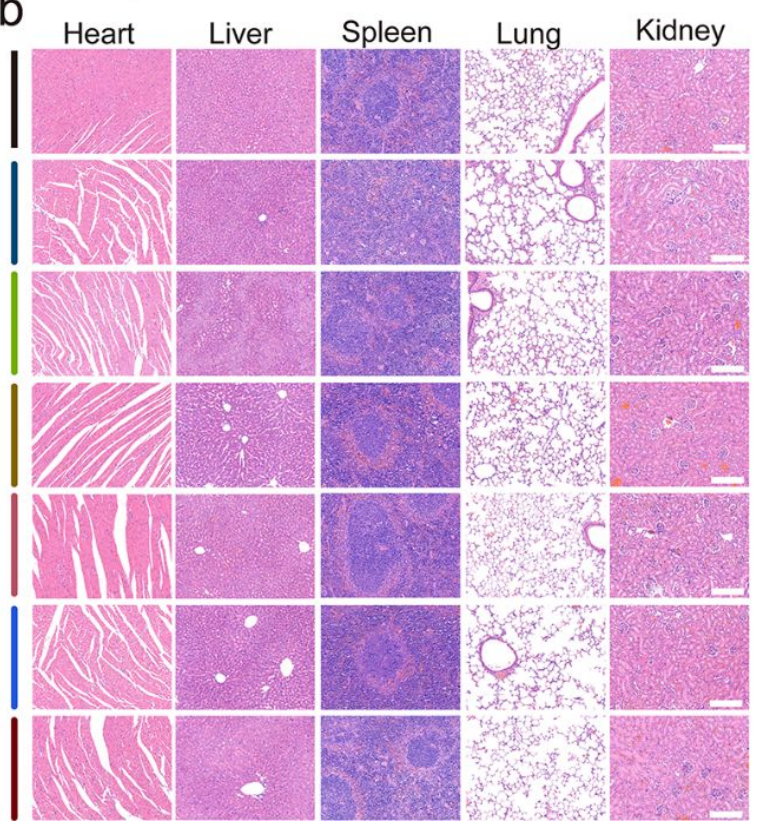

C
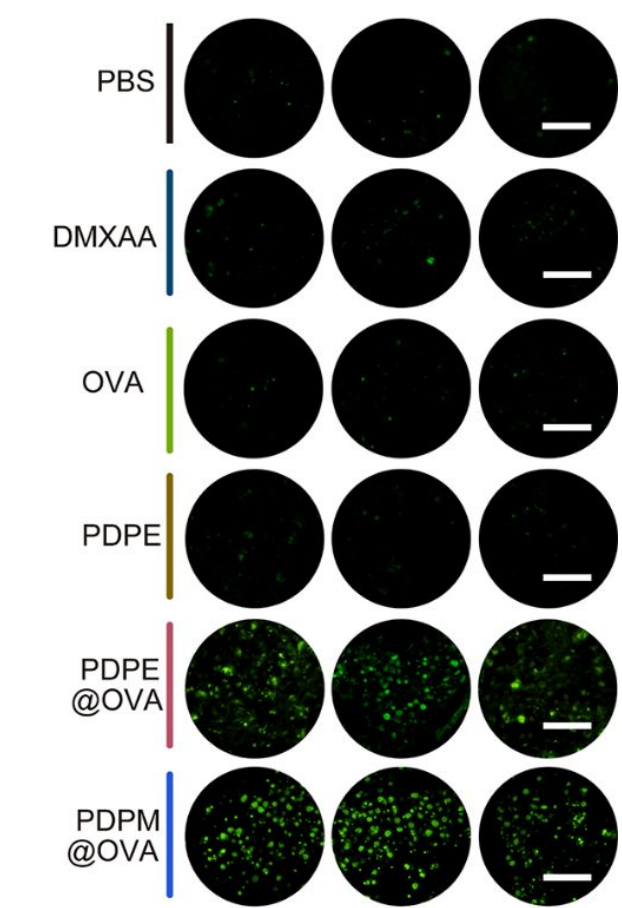

Man-PDPM @OVA

Figure S11. Immunofluorescence assay of tumors and biosafety assay of the

nanovaccines. (a) Body weight of vaccinated mice was measured over time; (b) H\&E analysis of major organs at the end of antitumor study (scale bar $=100 \mu \mathrm{m}$ ); (c) TUNEL staining of tumor sections determined at the end of antitumor study (scale bar $=50 \mu \mathrm{m})$. 

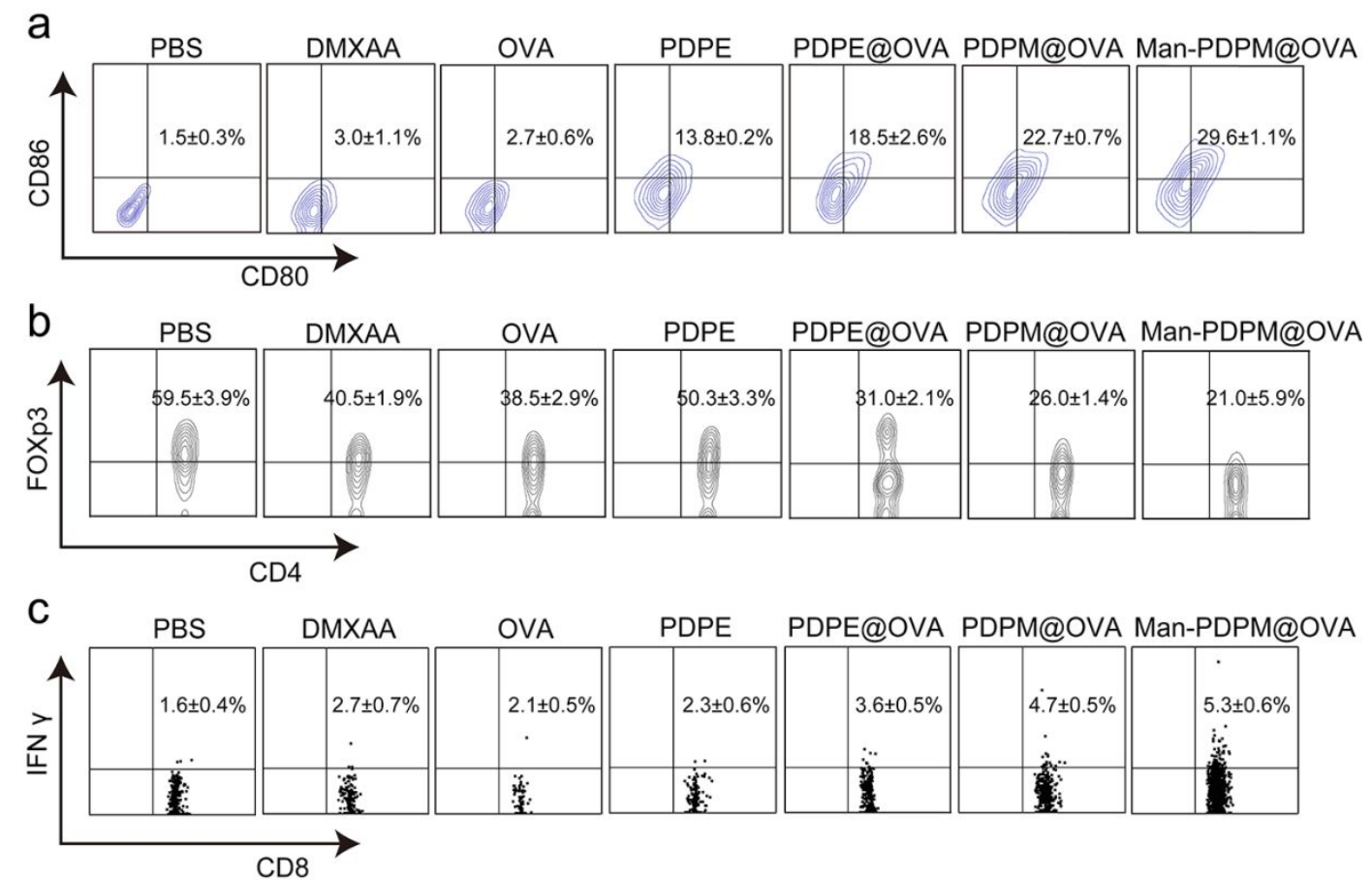

Figure S12. Immunoassay of the 4T1 tumor-bearing BALB/c mice after vaccination. (a) Counter plots of DC maturation stimulated by nanovaccines using flow cytometry. The lymph nodes were resected and digested into single cell suspension, following with CD11c-FITC, CD80-PE, CD86-PE-Cy7 staining; (b) The frequency of Tregs in tumor tissues on day 7 after various treatments; (c) Representative scatter plot of IFN- $\gamma^{+} \mathrm{CD} 8^{+}$effector T cells in the tumor tissues (gated on $\mathrm{CD} 45^{+} \mathrm{CD} 3^{+} \mathrm{CD} 8^{+}$). 
a

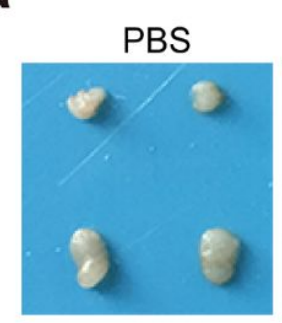

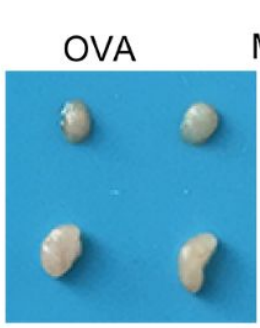

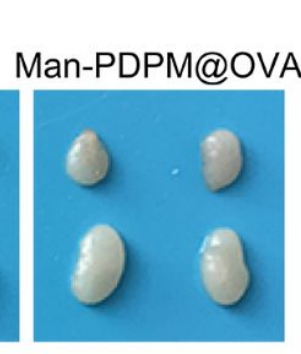

b

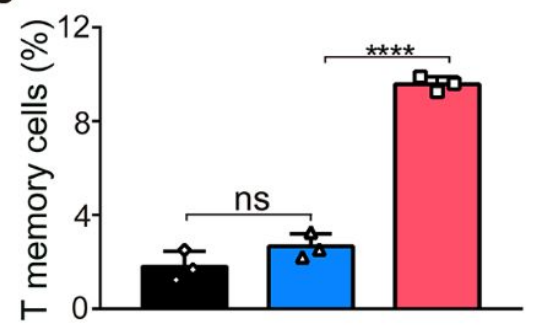

Figure S13. Prophylactic effect of the nanovaccine. (a) Representative photographs of the lymph nodes within mice collected on day-21 post the third vaccination. (b) The frequency of memory $\mathrm{T}$ cells $\left(\mathrm{CD} 8^{+} \mathrm{CD} 44^{+} \mathrm{CD} 127^{+}\right)$in the spleen after three-round vaccination. 

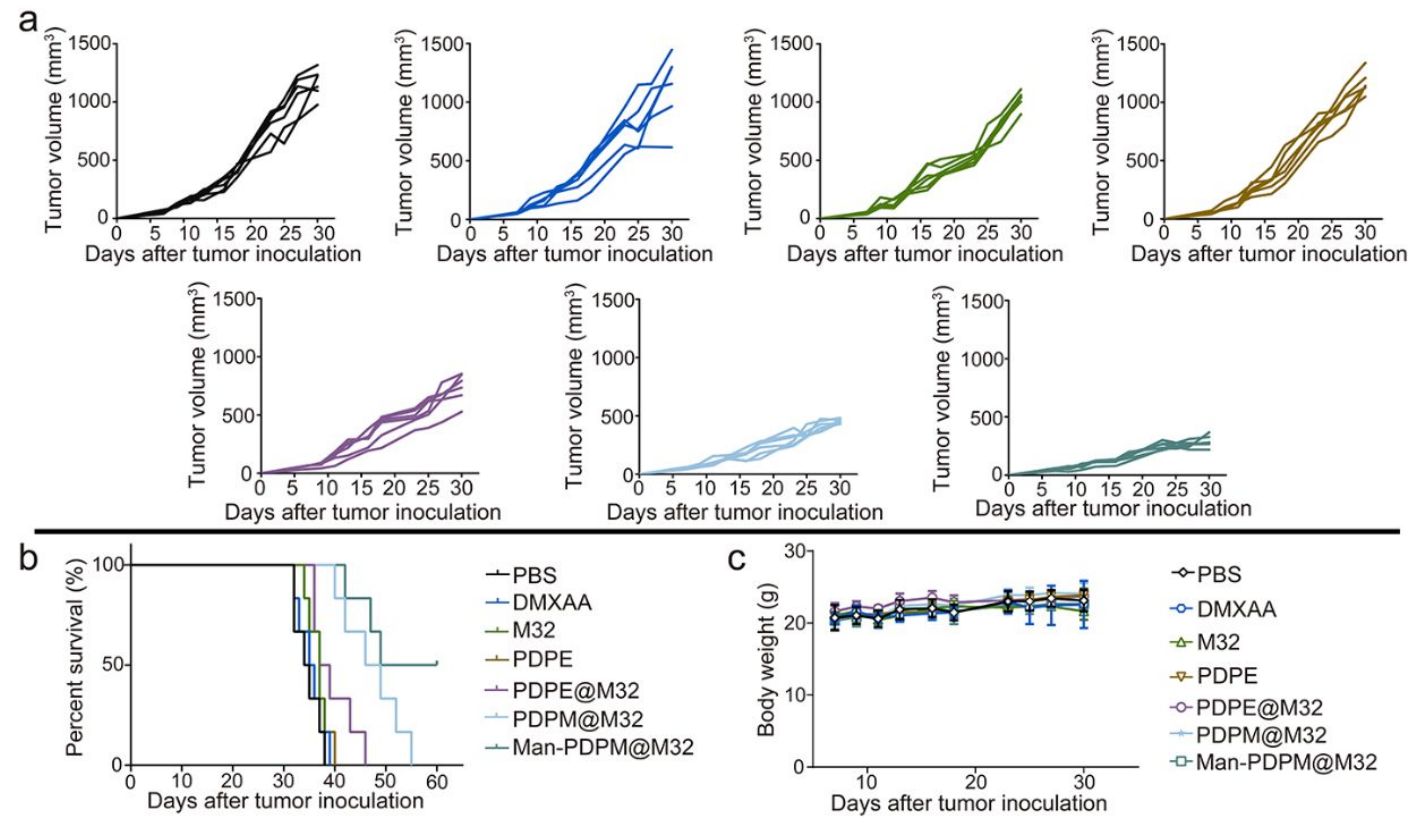

Figure S14. Antitumor efficacy of the neoantigen-loaded nanovaccines in 4T1

tumor models. (a) Individual tumor growth curve monitored over time with mice receiving desired treatments; (b) Survival curve of the 4T1 tumor-bearing BALB/c mice with various treatments. (c) Body weight change of the tumor bearing mice monitored during the antitumor study. 


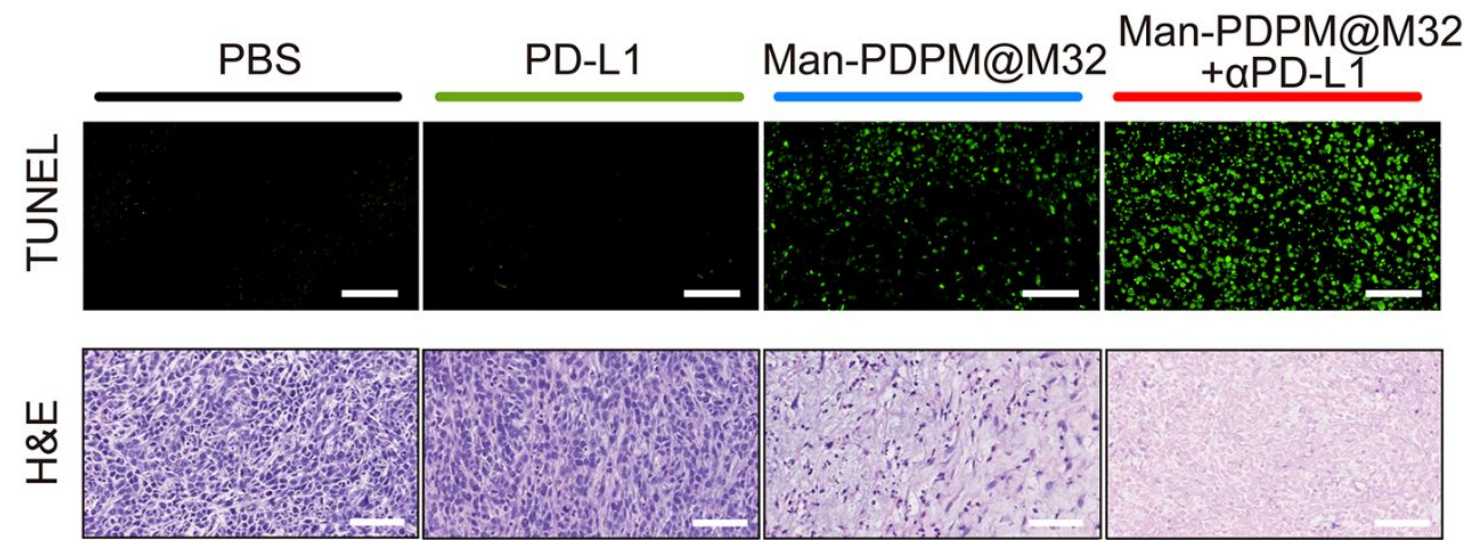

Figure 15. TUNEL and H\&E staining of the tumor sections at the end of combination therapy (scale $\operatorname{bar}=50 \mu \mathrm{m})$ 